\title{
Cost-effectiveness analysis of two kinds of bladder cancer urinary diversion: Studer versus Bricker
}

\author{
Weipu Mao ${ }^{1,2 \#}$, Jinbo Xie ${ }^{2 \#}$, Yuan $\mathrm{Wu}^{2 \#}$, Zonglin $\mathrm{Wu}^{1}$, Keyi Wang ${ }^{2}$, Heng Shi ${ }^{2}$, Hui Zhang ${ }^{3}$, Bo Peng ${ }^{1,2}$, \\ Jiang Geng ${ }^{2}$
}

${ }^{1}$ Department of Urology, People's Hospital of Putuo District, Shanghai 200060, China; ${ }^{2}$ Department of Urology, ${ }^{3}$ Department of Anesthesiology, Shanghai Tenth People's Hospital, School of Medicine, Tongji University, Shanghai 200072, China

Contributions: (I) Conception and design: W Mao, B Peng, J Geng; (II) Administrative support: Y Wu, Z Wu; (III) Provision of study materials or patients: Y Wu, K Wang, H Shi; (IV) Collection and assembly of data: W Mao, J Xie; (V) Data analysis and interpretation: W Mao, J Xie, H Zhang; (VI) Manuscript writing: All authors; (VII) Final approval of manuscript: All authors.

\#These authors contributed equally to this work.

Correspondence to: Jiang Geng; Bo Peng. Department of Urology, Shanghai Tenth People's Hospital, School of Medicine, Tongii University, No. 301, Yanchang Road, Jing'an District, Shanghai 200072, China. Email: gengjiangsn@sina.com; pengbo6908@163.com.

Background: The purpose of our study was to evaluated the cost-effectiveness of two bladder cancer (BCa) urinary diversions: Studer and Bricker.

Methods: The study included 44 patients with Studer and 40 patients with Bricker. Collected and analyzed the patient's basic characteristics, health care costs, and prognosis survival. The quality-adjusted life-year (QALY) were calculated and verified by the European Organization for Research and Treatment of Cancer Quality of Life Questionnaire Core-30 (EORTC QLQ-C30, Version 3, Chinese version). Cost-effectiveness depends on the incremental cost per QALY. The incremental cost-effectiveness ratio (ICER) was determined using the cost/QALY.

Results: We found the average total cost of the Studer group was $\$ 7,173.7 \pm 1,390.8$, and the Bricker group was $\$ 6,545.2 \pm 1,458.4$. There were significant differences in hospitalization time, total hospitalization expenses, bed cost, comprehensive medical service charge and drugs cost (all $\mathrm{P}<0.05)$. The hospitalization time, total hospitalization expenses, bed cost, comprehensive medical service charge, surgical treatment cost and drugs cost in Studer group were higher than those in Bricker group, while there was no significant difference in postoperative complications between the two groups $(\mathrm{P}=0.858)$. The ICER of Studer group and Bricker group were \$8,535.6 $\pm 2,027.6 / \mathrm{QALY}$ and $\$ 11,158.2 \pm 2,944.9 / \mathrm{QALY}$, respectively. The ICER of Studer group over Bricker group was \$2,514.0/QALY.

Conclusions: We found the Studer group had higher hospitalization time, total hospitalization expenses, bed cost, comprehensive medical service charge, surgical treatment cost, and drugs cost than the Bricker group, but the Studer group had a higher ICER than the Bricker group.

Keywords: Bladder cancer urinary diversion (BCa urinary diversion); Studer; Bricker; quality-adjusted life-year (QALY); incremental cost-effectiveness ratio (ICER)

Submitted Dec 05, 2019. Accepted for publication Mar 12, 2020.

doi: $10.21037 /$ tau.2020.03.46

View this article at: http://dx.doi.org/10.21037/tau.2020.03.46 


\section{Introduction}

Bladder cancer $(\mathrm{BCa})$ is the second most common cancer after prostate cancer in the urinary system. In the United States, 80,470 new cases of BCa were estimated in 2019, accounting for $4.6 \%$ of all new cancers, approximately 17,670 deaths, accounting for $2.9 \%$ of all deaths (1). According to the depth of tumour invasion, BCa can be divided into non-muscle invasive bladder cancer (NMIBC) and muscle-invasive bladder cancer (MIBC) (2). Approximately $70 \%$ of $\mathrm{BCa}$ is NIMBC, which has the characteristics of repeated recurrence, the recurrence rate is as high as $30-80 \%(3,4)$. BCa patients and families often bear a huge economic and psychological burden.

The treatment of $\mathrm{BCa}$ patients includes surgery, radiotherapy, chemotherapy and other treatments (5). Surgery is the preferred treatment for patients with BCa. For NMIBC patients, transurethral resection of bladder tumours (TURBT) combined with postoperative intravesical infusion chemotherapy is the main treatment (6), but for high-risk NMIBC patients, early radical cystectomy (RC) is also necessary (7). RC combined with pelvic lymph node dissection is the standard surgical method for MIBC patients, and the choice of postoperative urinary diversion is particularly important $(8,9)$. There are many types of urinary diversion after RC, such as ureteral skin ostomy, Bricker ileal cystectomy, Studer in situ cystectomy and so on (10-12).

To date, no study has been conducted to compare the cost-effectiveness of Studer and Bricker urinary diversions. In this study, we compared the total hospitalization costs of the two surgical methods for BCa by collecting various indicators of patients and used the incremental cost-benefit ratio (ICER) to analyze the cost-effectiveness.

\section{Methods}

\section{Patients information}

In this study, we retrospectively obtained RC patients medical and financial information from the hospitalization medical and financial records department between January 2009 and July 2015 at the department of urology, Shanghai Tenth People's Hospital of Tongji University (Shanghai, China). All patients were diagnosed with $\mathrm{BCa}$ according to the 2002 American Joint Committee on Cancer/Union for International Cancer Control Tumour, Lymph Node and Metastasis (TNM) staging system. The study was approved by the Ethics Committee of Shanghai Tenth People's
Hospital of Tongii University (SHSY-IEC-4.1/19-120/01). All patients with $\mathrm{BCa}$ who underwent $\mathrm{RC}$ had a detailed understanding of the differences between the two surgical methods before operation and the mode of operation was depend on the patients willing. All patients or their relatives received written informed consent. The methodology of this study followed the standards outlined in the Declaration of Helsinki

The inclusion criteria in our study were as follows: (I) older than 18 years of age; (II) follow-up time greater than 6 months; (III) no previous history of mental illness and severe cognitive impairment; (IV) underwent Studer or Bricker.

The exclusion criteria were as follows: (I) patients with incomplete hospitalization information; (II) follow-up lost patients; (III) incomplete questionnaire core; (IV) associated with other diseases that seriously affect health-related quality of life.

According to the above conditions, 84 patients with RC and urinary diversion in our department of urology from January 2009 to July 2015 were selected, including 44 cases underwent Studer and 40 cases underwent Bricker. All patients were followed up until June 2019.

\section{Study variables}

In this study, we collected demographic data for patients including sex, age, body mass index (BMI), comprehensive complication index (CCI), TNM stage, grade, and prognostic survival time. Hospitalization information includes total hospitalization expenses, bed cost, comprehensive medical service (general medical service cost, general treatment operation cast, nursing care and other costs), diagnosis costs (pathological diagnosis cost, laboratory diagnostic cost, imaging diagnosis cost and clinical diagnosis project cost), surgical treatment costs (anesthesia and surgery), drugs fees, surgical equipment and medical materials, and other treatment expenses. All fees were converted into US dollars (\$) at their value in September 2019.

The quality-adjusted life-year (QALY) were calculated and verified by the European Organization for Research and Treatment of Cancer Quality of Life Questionnaire Core30 (EORTC QLQ-C30, Version 3, Chinese version) (13). The EORTC QLQ-C30 Chinese version of the scale contains 30 entries in 15 fields. They were divided into 5 functional areas (physical functioning, role functioning, cognition functioning, emotional functioning and social 
functioning), 3 symptom areas (fatigue, pain, nausea/ vomiting), 6 single entries and 1 overall quality of life area. The overall quality of life field scored 1-7 points, other field entries scored 1-4 points, and the QALY score was between $0-1$ through linear conversion. The higher the score was, the higher quality life patient had.

Cost-effectiveness depended on the incremental cost per QALY. The incremental cost-effectiveness ratio (ICER) was namely the result of cost/QALY.

$$
I C E R=\frac{C O S T_{\text {Studer }}-C O S T_{\text {Bricker }}}{Q A L Y_{\text {Studer }}-Q A L Y_{\text {Bricker }}}
$$

\section{Statistical analysis}

The analysis of variance was used to compare the different continuous variables of urinary diversion between the two groups, including average and standard deviation. Chisquare test was used to determine the baseline variables for both groups of patients. In addition, patients with Studer and Bricker were matched with 1:1 propensity score matching (PSM) for sex, diagnosis age, BMI, CCI, TNM stage and grade to reduce the possibility of selection bias. All statistical analyses were performed using the Social Science Software package (version 20.0; SPSS Inc, Chicago, IL, USA). $\mathrm{P}<0.05$ was considered to be statistically significant.

\section{Results}

\section{Demographic and clinical characteristics of the patients}

In our study, we screened 84 eligible patients from January 2009 to July 2015, including 44 in the Studer group and 40 in the Bricker group. Table 1 shows the clinical baseline characteristics of the two groups of patients. In the whole cohort, there were 77 males and 7 females, including 42 males and 2 females in the Studer group, 35 males and 5 females in the Bricker group. The average age of the Studer group was lower than that of the Bricker group $(60.7 \pm 6.7$ vs. $65.8 \pm 7.0 ; \mathrm{P}=0.001)$. There was significant difference in hospitalization time between the two groups (Studer: 39.0 \pm 9.6 days, Bricker: $29.4 \pm 9.4$ days; $\mathrm{P}<0.001)$ and the hospitalization time in the Studer group was higher than that in the Bricker group. In addition, there were no significant differences in BMI, sex, T-stage, N-stage, M-stage, grade and survival time between the two groups. After PSM, 20 patients in the Studer and Bricker groups were selected.
There was a significant difference in hospitalization time between the two groups, and the length of hospitalization time in Studer group $(41.7 \pm 10.1$ days $)$ was significantly longer than that in Bricker group (25.8 \pm 8.7 days).

\section{Comparison of postoperative complications}

In addition, we performed postoperative follow-up on all patients (Table 2). We found that there were 6 patients with postoperative complications both in the Studer group and Bricker group, and there was no significant difference in postoperative complications between the two groups $(\mathrm{P}=0.858)$. The number of cases of difficulty urinating, ureteral anastomotic stricture, neobladder urethral anastomotic stricture, urethral tumor recurrence and incomplete intestinal obstruction were 1,2, 0, 2 and 0 in Studer group, while in Bricker group were 0, 2, 0, 1, 3 .

\section{Hospitalization costs for two surgical techniques}

The costs of the two surgical techniques were summarized in Table 3. The average total hospitalization cost in the Studer group was $\$ 7,173.7 \pm 1,390.8$, and the average total hospitalization cost in the Bricker group was $\$ 6,545.2 \pm 1,458.4$. There were significant differences between the two groups in total hospitalization cost, bed cost, comprehensive medical cost, drug cost and other treatment cost, but no difference in diagnosis cost, surgical treatment cost and surgical equipment and medical material cost. Whether before PSM or after PSM, the total hospitalization expenses, bed cost, comprehensive medical cost, total cost of surgical treatment, drug cost, surgical equipment and medical material cost of Studer group were higher than those of Bricker group.

\section{Cost-utility analysis}

The QALY of the Studer group and the Bricker group were $0.85 \pm 0.09$ and $0.60 \pm 0.08$ respectively $(\mathrm{P}<0.001)$. Before PSM, the cost per QALY for the Studer group was $\$ 8,535.6 \pm 2,027.6$, while that the Bricker group was $\$ 11,158.2 \pm 2,944.9(\mathrm{P}=0.001)$, and the cost per QALY in Studer group was $\$ 8,694.7 \pm 2,160.4$, while that in Bricker group was $\$ 10,815.5 \pm 2,361.0(\mathrm{P}=0.001)$ after PSM. Compared with the Bricker group, the Studer group saved \$2,622.6 per QALY before PSM and \$2,120.2 per QALY after PSM. The ICER of the Studer group was $\$ 2,514.0$ before PSM and \$2,971.7 after PSM (Table 4). 
Table 1 Baseline characteristics of the study population

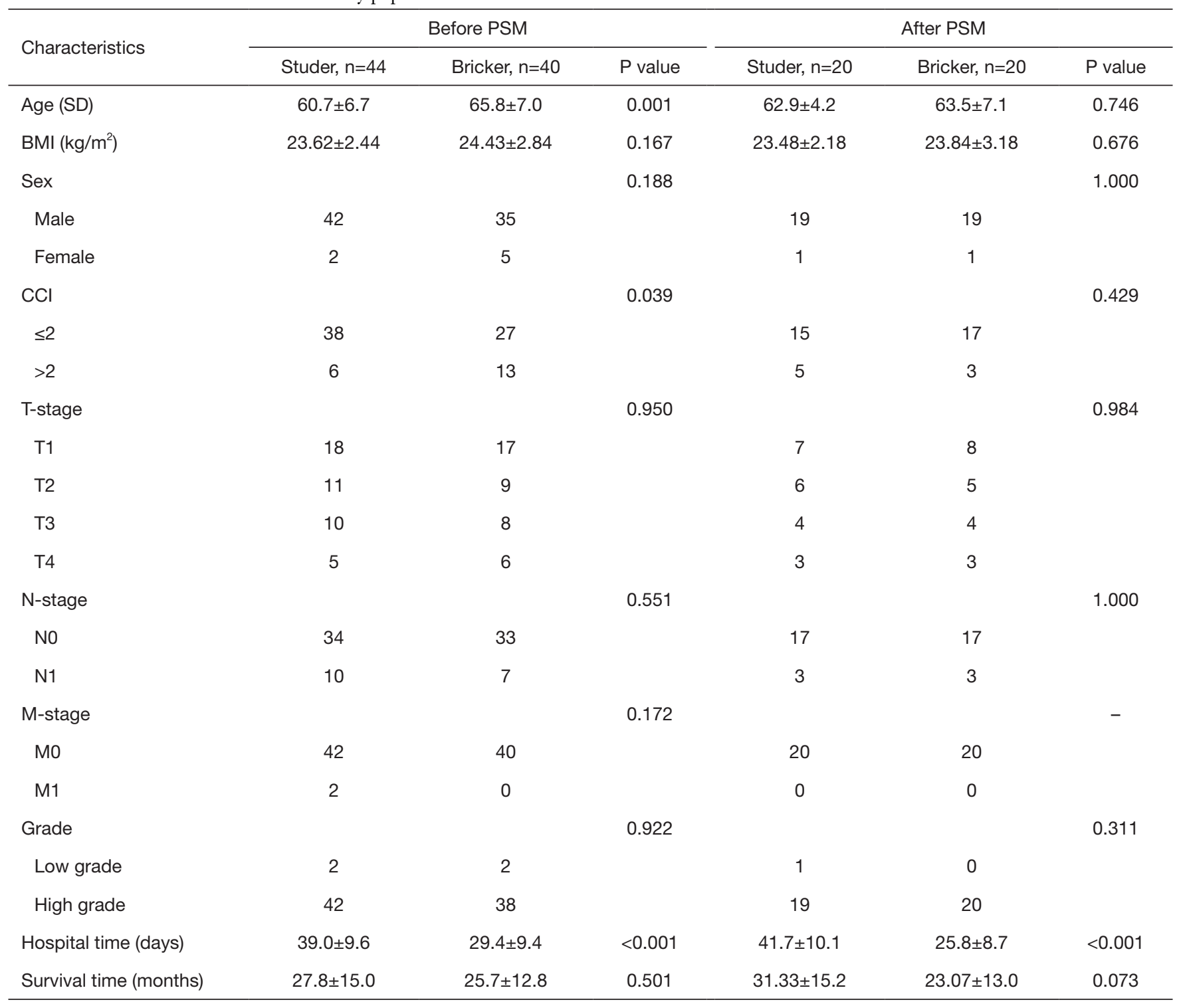

PSM, propensity score matching; BMI, body mass index; $\mathrm{CCl}$, comprehensive complication index.

Table 2 Comparison of postoperative complications between two groups

\begin{tabular}{lcc}
\hline Characteristics & Studer $(\mathrm{n}=44)$ & Bricker $(\mathrm{n}=40)$ \\
\hline Difficulty urinating & $1(2.3 \%)$ & - \\
Ureteral anastomotic stricture & $2(4.5 \%)$ & $2(5.0 \%)$ \\
Neobladder urethral anastomotic stricture & $1(2.3 \%)$ & - \\
Urethral tumor recurrence & $2(4.5 \%)$ & $1(2.5 \%)$ \\
Incomplete intestinal obstruction & $6(13.6 \%)$ & $3(7.5 \%)$ \\
Total & & 6.858 \\
\hline
\end{tabular}


Table 3 Hospital costs for two surgical techniques

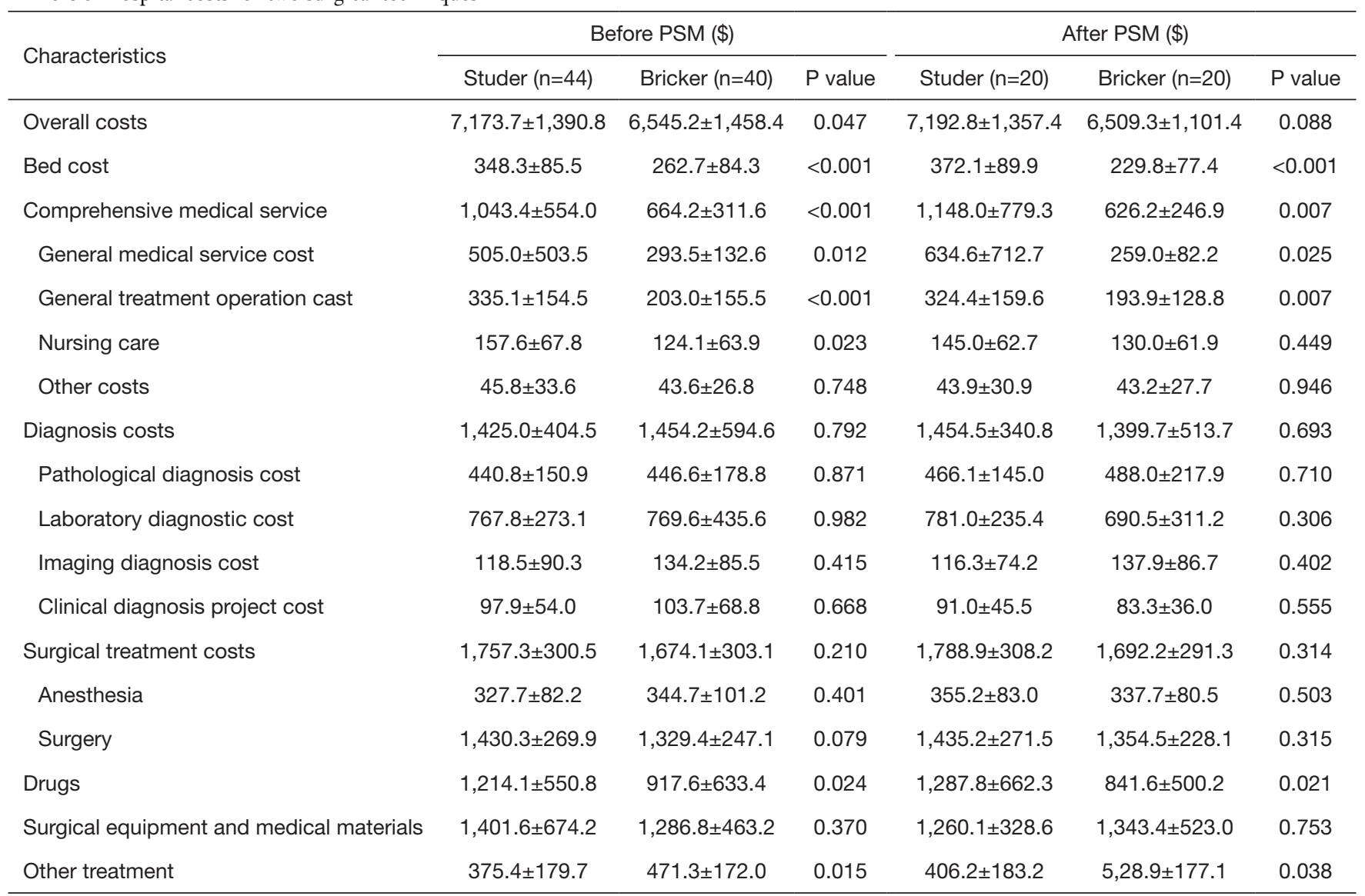

PSM, propensity score matching.

Table 4 ICER between Studer and Bricker groups

\begin{tabular}{|c|c|c|c|c|c|c|}
\hline Characteristics & \multicolumn{3}{|c|}{ Before PSM } & \multicolumn{3}{|c|}{ After PSM } \\
\hline QALY & $0.85 \pm 0.09$ & $0.60 \pm 0.08$ & $<0.001$ & $0.84 \pm 0.10$ & $0.61 \pm 0.07$ & $<0.001$ \\
\hline Cost/QALY & $\$ 8,535.6 \pm 2,027.6$ & $\$ 11,158.2 \pm 2,944.9$ & 0.001 & $\$ 8,694.7 \pm 2,160.4$ & $\$ 10,815.5 \pm 2,361.0$ & 0.001 \\
\hline ICER & $\$ 2,514.0$ & & & $\$ 2,971.7$ & & \\
\hline
\end{tabular}

PSM, propensity score matching; QALY, quality-adjusted life-year; ICER, incremental cost-effectiveness ratio.

\section{Discussion}

For MIBC, even with standard RC combined with chemotherapy, the 5 -year survival rate was only $50 \%(9,14)$. The treatment of advanced $\mathrm{BCa}$ poses a huge clinical and economic burdens on the health care system (15). RC was one of the most important methods for the treatment of invasive BCa clinically (16). The choice of urinary diversion after RC was crucial. Since the successful experience of
Bricker's report of ileal cystectomy in the 1950s, various controlled and uncontrollable bladder replacement and urinary diversions treatments have been introduced.

This study determined the cost-effectiveness of two urinary diversions (Studer and Bricker) after total cystectomy by combining the data currently collected. Our results show that compared with Bricker group, Studer group has higher hospitalization time, total hospitalization cost, bed cost, comprehensive medical service cost, surgical 
treatment cost and drug cost, but there was no significant difference in postoperative complications between the two groups. The ICER of Studer group and Bricker group were $\$ 8,535.6 \pm 2,027.6 /$ QALY and $\$ 8,694.7 \pm 2,160.4 /$ QALY, respectively. The Studer group has an ICER of \$2,514.0/ QALY for the Bricker group. Similar result was obtained after 1:1 PSM for sex, age, BMI, TNM stage and grade, indicating that although Studer spends more cost, Studer can achieve better quality of life and had better costeffectiveness. Studer is a better choice for young patients with a good family income.

The higher total hospitalization cost in the Studer group may be associated with the complicated surgical procedures, surgical equipment consumables, and the longer duration of hospitalization. Previous studies have proved that hospitalization time was closely related to hospitalization costs and reducing the duration of hospitalization can significantly reduce the cost of RC (17). In addition, due to the extension of hospitalization time, the cost of comprehensive medical services and drugs will also increase, which will also result to a higher total hospitalization expense in the Studer group (18).

According to the statistics of the postoperative complications, we found that there was no significant difference in the total postoperative complications between the two groups. The incidence of difficulty urinating $(2.3 \%$ vs. $0 \%)$ and neobladder urethral anastomotic stricture $(2.3 \%$ vs. $0 \%)$ in Studer group was higher than that in Bricker group, while the incidence of ureteral anastomotic stricture ( $4.5 \%$ vs. $5.0 \%)$, urethral tumor recurrence ( $0 \%$ vs. $2.5 \%)$ and incomplete intestinal obstruction $(4.5 \%$ vs. $7.5 \%)$ was lower. This may be related to the younger patients in the Studer group, the better bowel function, the continuous flushing of urine and the anti-cancer effect of mucin secreted by the neobladder ileum mucosa $(19,20)$.

Bricker ileal cystectomy was one of the most common surgical methods for uncontrollable urinary diversion (21). The operation is relatively simple, but the patients need abdominal wall colostomy, wearing a urine collection bags for life and the quality of life after surgery is poor. Compared with Bricker, patients with Studer do not require abdominal wall colostomy and the quality of life after surgery is high, which gradually becomes the preferred procedure for urinary diversion (22-24).

The choice of urinary diversion after RC for bladder tumor needs to be made according to the nature and location of the tumor, the age of the patient, the tolerance of operation, the general situation and the willingness of patient (25). At the same time, we should also take into account the operator's proficiency in the selected operation in order to achieve the purpose of clinically curing the tumor and guarantee of quality of life as much as possible. In addition, careful management of the perioperative period and close follow-up after operation are also the key to the successful surgery.

Our study also has some limitations. First, this study is a retrospective study with its own limitations. Second, for patients readmitted due to postoperative complications, we cannot obtain the exact follow-up costs. Although we performed PSM on patient's sex, age, BMI, TNM stage and grade, patient preferences and family financial burden also affected patient choice, and more cases needed for prospective studies.

Our study compared the economic evaluation results of Studer and Bricker. We found that although Studer has higher cost than Bricker, Studer can get higher quality of life and higher ICER.

\section{Acknowledgments}

The authors are grateful for the invaluable support and useful discussions with other members of the urological department.

Funding: This work was supported by grant from the National Natural Science Foundation of China (81001134) to Jiang Geng, Shanghai Science and Technology Commission (Grant No.18140900302) and the National Natural Science Foundation of China (31670772) to Bo Peng.

\section{Footnote}

Conflicts of Interest: All authors have completed the ICMJE uniform disclosure form (available at http://dx.doi. org/10.21037/tau.2020.03.46). The authors have no conflicts of interest to declare.

Ethical Statement: The authors are accountable for all aspects of the work in ensuring that questions related to the accuracy or integrity of any part of the work are appropriately investigated and resolved. The study was in line with the Helsinki Declaration and approved by the Ethics Committee at the Tenth People's Hospital of Shanghai (SHSY-IEC-4.1/19-120/01). The study outcomes will not affect the future management of the patients. Informed consent was obtained from the controls and patients or their relatives. 
Open Access Statement: This is an Open Access article distributed in accordance with the Creative Commons Attribution-NonCommercial-NoDerivs 4.0 International License (CC BY-NC-ND 4.0), which permits the noncommercial replication and distribution of the article with the strict proviso that no changes or edits are made and the original work is properly cited (including links to both the formal publication through the relevant DOI and the license). See: https://creativecommons.org/licenses/by-nc-nd/4.0/.

\section{References}

1. Siegel RL, Miller KD, Jemal A. Cancer statistics, 2019. CA Cancer J Clin 2019;69:7-34.

2. Czerniak B, Dinney C, McConkey D. Origins of Bladder Cancer. Annu Rev Pathol 2016;11:149-74.

3. Burger M, Catto JW, Dalbagni G, et al. Epidemiology and risk factors of urothelial bladder cancer. Eur Urol 2013;63:234-41.

4. Mao W, Huang X, Wang L, et al. Circular RNA hsa_ circ_0068871 regulates FGFR3 expression and activates STAT3 by targeting miR-181a-5p to promote bladder cancer progression. J Exp Clin Cancer Res 2019;38:169.

5. Babjuk M, Burger M, Comperat EM, et al. European Association of Urology Guidelines on Non-muscleinvasive Bladder Cancer (TaT1 and Carcinoma In Situ) 2019 Update. Eur Urol 2019;76:639-57.

6. Babjuk M, Oosterlinck W, Sylvester R, et al. EAU guidelines on non-muscle-invasive urothelial carcinoma of the bladder. Eur Urol 2008;54:303-14.

7. Hautmann RE, Volkmer BG, Gust K. Quantification of the survival benefit of early versus deferred cystectomy in high-risk non-muscle invasive bladder cancer (T1 G3). World J Urol 2009;27:347-51.

8. Gupta NP, Goel R, Hemal AK, et al. Radical cystectomy in septuagenarian patients with bladder cancer. Int Urol Nephrol 2004;36:353-8.

9. Alfred Witjes J, Lebret T, Comperat EM, et al. Updated 2016 EAU Guidelines on Muscle-invasive and Metastatic Bladder Cancer. Eur Urol 2017;71:462-75.

10. Stenzl A, Cowan NC, De Santis M, et al. The updated EAU guidelines on muscle-invasive and metastatic bladder cancer. Eur Urol 2009;55:815-25.

11. Bricker EM. Bladder substitution after pelvic evisceration. Surg Clin North Am 1950;30:1511-21.

12. Studer UE, Zingg EJ. Ileal orthotopic bladder substitutes. What we have learned from 12 years' experience with 200 patients. Urol Clin North Am 1997;24:781-93.
13. Chun ZF, Mitchell JE, Li KA, et al. The Prevalence Of Anorexia-Nervosa And Bulimia-Nervosa among Freshman Medical-College Students In China. International Journal of Eating Disorders 1992;12:209-14.

14. Hussain SA, James ND. The systemic treatment of advanced and metastatic bladder cancer. Lancet Oncol 2003;4:489-97.

15. Kamat AM, Hahn NM, Efstathiou JA, et al. Bladder cancer. Lancet 2016;388:2796-810.

16. Nieuwenhuijzen JA, de Vries RR, Bex A, et al. Urinary diversions after cystectomy: the association of clinical factors, complications and functional results of four different diversions. Eur Urol 2008;53:834-42; discussion 842-4.

17. Lee R, Ng CK, Shariat SF, et al. The economics of robotic cystectomy: cost comparison of open versus robotic cystectomy. BJU Int 2011;108:1886-92.

18. Michels CTJ, Wijburg CJ, Leijte E, et al. A costeffectiveness modeling study of robot-assisted (RARC) versus open radical cystectomy (ORC) for bladder cancer to inform future research. Eur Urol Focus 2019;5:1058-65.

19. Freeman JA, Tarter TA, Esrig D, et al. Urethral recurrence in patients with orthotopic ileal neobladders. J Urol 1996;156:1615-9.

20. Studer UE, Ackermann D, Casanova GA, et al. 3 Years Experience with an Ileal Low-Pressure Bladder Substitute. Br J Urol 1989;63:43-52.

21. Nabi G, Yong SM, Ong E, et al. Is orthotopic bladder replacement the new gold standard? Evidence from a systematic review. J Urol 2005;174:21-8.

22. Gerharz EW. Is there any evidence that one continent diversion is any better than any other or than ileal conduit? Curr Opin Urol 2007;17:402-7.

23. Parekh DJ, Gilbert WB, Koch MO, et al. Continent urinary reconstruction versus ileal conduit: A contemporary single-institution comparison of perioperative morbidity and mortality. Urology 2000;55:852-5.

24. Hautmann RE. Urinary diversion: Ileal conduit to neobladder. J Urol 2003;169:834-42.

25. Nezhat CR, Nezhat F, Luciano AA, et al. editors. Operative Gynecologic Laparoscopy: Principles and Techniques. McGraw-Hill, 1995, 337 pages.

Cite this article as: Mao W, Xie J, Wu Y, Wu Z, Wang K, Shi H, Zhang H, Peng B, Geng J. Cost-effectiveness analysis of two kinds of bladder cancer urinary diversion: Studer versus Bricker. Transl Androl Urol 2020;9(3):1113-1119. doi:10.21037/ tau.2020.03.46 